\title{
Dependencia serial de largo plazo en el índice bursátil chileno, a través del coeficiente de Hurst y Hurst ajustado

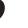

\author{
Christian Acuña-Opazo and Alejandro Álvarez-Marín \\ Departamento de Ingenieria Industrial, Universidad de La Serena, \\ La Serena, Chile
}

Long term serial dependence 
JEFAS

22,42

Findings - The results demonstrate the presence of long-term memory in the Chilean stock market, determined by stock index in two scales, daily and quarterly, which also corroborates results obtained by other authors, confirming the use of the methodology Range Re-scalded for the identification and determination of long-term memory in a time series.

Originality/value - This study will allow future researchers to perform similar analyzes in other markets, providing a new approach when analyzing the long-term memory and the factors that affect it.

Keywords Efficient markets, Fractal markets, Hurst exponent, Stock index

Paper type Research paper

\section{Introducción}

Durante las últimas décadas, muchos países de Europa, Asia y América latina han experimentado gran dinamismo en sus mercados a través de la inversión extranjera directa (IED) originado, entre otros factores, por la inversión en infraestructura e inversión en desarrollo tecnológico y, especialmente, por la explotación de recursos naturales en América latina, por parte de países desarrollados como Estados Unidos, China, Canadá y España, por mencionar algunos (CEPAL, 2011). Lo anterior se complementa con la visión que han tenido distintos inversionistas internacionales, luego de la crisis experimentada por Estados Unidos en el año 2008, transformándose América latina en un escenario de atractiva generación de riqueza (FMI, 2010). Esto ha provocado un mayor dinamismo en los mercados de inversiones, mercado de capitales o bursátiles de los diferentes países, en adelante mercado bursátil. En este sentido, mayor interés ha cobrado el estudio y análisis de los mercados de capitales y su comportamiento a través de sus diferentes índices bursátiles, donde Chile no está ajeno a dicho escenario.

El estudio del comportamiento de los índices bursátiles, específicamente, el comportamiento de sus rendimientos es fundamental para comprender el desempeño de los mercados bursátiles. Por esto se hace necesario investigar el cumplimiento de los supuestos en los cuales se fundamentan la mayoría de modelos de valoración de activos, como por ejemplo el Capital Asset Pricing Model (CAPM). Gran parte de los modelos utilizados para el análisis del comportamiento de este tipo de mercados, y por tanto de sus variables, como el precio de las acciones, se basan en la Hipótesis de los Mercados Eficientes (HME) (Restrepo y Velásquez, 2011), donde se precisa que los precios de equilibrio de mercado presentan de manera automática la información histórica y nueva (Fama, 1965), y que los agentes participantes de dichos mercados se comportan racionalmente y con aversión al riesgo (Fama y French, 1988).

Durante muchos años, las pruebas de comportamiento aleatorio han sido la herramienta de evaluación de la eficiencia del mercado y, por tanto, la validez de los modelos empleados, los que desde la década del 60 respaldaban el paradigma actual; sin embargo, y en el escenario de la nueva economía, el paradigma de la complejidad ha tomado fuerza, desarrollándose una nueva corriente con el objeto de buscar comportamientos complejos en los mercados bursátiles, y en este sentido, posibilitar una nueva configuración de las herramientas que permitan predecir el comportamiento de los rendimientos futuros de los activos. A este respecto, varios han sido los estudios que han evidenciado que el comportamiento de los precios de acciones, no sigue un camino aleatorio (Lo y MacKinley, 1988; Davies y Harte, 1987; Fama y French, 1988), lo que permite pensar en la existencia de dependencia serial de largo plazo o memoria de largo plazo en las series de valores bursátiles. Este fenómeno de dependencia serial financiera significa que los rendimientos de un activo hoy pueden tener una incidencia sobre los rendimientos futuros. Peters (1989) explica este fenómeno a través del concepto de 
“sentimiento del mercado" (Rodríguez, 2011), y que puede provocar que una tendencia se mantenga en el mercado aun cuando no se introduzcan nueva información o noticias en dicho mercado.

Mandelbrot (Mandelbrot y Wallis, 1969; Mandelbrot, 1972), fue uno de los primeros investigadores en buscar dependencias en las series temporales económicas y financieras. A partir de ello, varios estudios han encontrado evidencia a favor de dicha dependencia serial (Peters, 1992; Soria y Zúñiga, 2004; Espinosa, 2007). Karuppiah y Los (2005) encontraron dependencia en ocho mercados asiáticos, mientras que otros autores lo hicieron en los retornos accionarios de Corea, Malasia, Singapur y Nueva Zelanda (Sadique y Silvapulle, 2001).

Considerando que muchos trabajos de carácter empírico apoyan la existencia de un grado de memoria de largo plazo en las series económicas y financieras de largo plazo, los métodos empleados para contrastar esta evidencia en muchos casos difieren. Ejemplo de lo anterior son los métodos planteados por Hurst, Hosking, Geweke y Porter-Hudak, Lo y Agiakloglou, entre otros (citados por Espinosa, 2007; León y Vivas, 2010).

Entre los diferentes estudios presentes en la literatura, algunos señalan la existencia de consecuencias, tanto prácticas como teóricas de la memoria de largo plazo; entre ellas, la violación de supuestos del movimiento browniano (Holton, 1992; León y Vivas, 2010; León y Reveiz, 2010) y sesgo de sobreestimación de parámetros utilizados para contrastar las hipótesis de caminata aleatoria de las series (Peters, 1994; León y Vivas, 2010; León y Reveiz, 2010).

Un punto común en las investigaciones sobre la memoria de largo plazo en un índice bursátil, es que la mayoría de los autores ha empleado el coeficiente de Hurst para contrastar la hipótesis de mercado eficiente. Sin embargo, pocos son los trabajos que han probado la existencia de esta memoria de largo plazo en las series financieras, por medio del índice bursátil chileno, específicamente IPSA, considerando los sesgos que presenta el coeficiente de Hurst para contrastar la hipótesis de mercado eficiente (Espinosa et al., 2005), memoria o dependencia de largo plazo (Espinosa, 2007; Soria y Zúñiga, 2004), y ninguno ha analizado la dependencia serial de largo plazo en el mercado bursátil de Chile por medio del coeficiente de Hurst, considerando la metodología de ajuste del coeficiente de Hurst propuesta por Anis y Lloyd (1976) o Peters (1994).

De acuerdo con los hallazgos de Mandelbrot (1972), Peters (1992; 1994), Bilel y Nadhem (2009) y León y Reveiz (2010), para series financieras de tiempo donde el volumen transado es propio de economías desarrolladas, y de Soria y Zúñiga, (2004), Espinosa et al. (2005) y Parisi et al. (2007). Para el mercado chileno, este trabajo propone analizar la serie financiera del IPSA en cuanto a encontrar indicios de memoria de largo plazo en la serie financiera por medio del cálculo del coeficiente de Hurst $(\mathrm{H})$ y Hurst ajustado $\left(\mathrm{H}_{\mathrm{adj}}\right)$ y de características de estructuras caóticas en las series en el mercado bursátil de Chile, específicamente a través del Índice de Precios Selectivo de Acciones (IPSA).

\section{Marco teórico}

A continuación se presentan los elementos teóricos relevantes que permitirán dar cumplimiento a los objetivos de esta investigación. En primer lugar, se enuncian algunas de las características de comportamiento presentes en los mercados bursátiles, analizadas por medio de la presencia de la Hipótesis de Mercado Eficiente (HME) e Hipótesis de Mercados Fractales (HMF). En segundo lugar, se describe el método de cálculo del coeficiente de Hurst y Hurst ajustado, por medio del procedimiento del Rango Reescalado (R/S). 
JEFAS

22,42

\section{Mercado eficiente y mercado fractal}

Para Fama (1965), un mercado de capitales eficiente es aquel en que la información procesada es eficiente; es decir, está libremente disponible para todos aquellos que participan en dicho mercado, por cuanto los valores de los precios observados en cualquier momento se basan en un correcto procesamiento de la información total disponible en dicho momento. Por esta razón, se entiende que los precios presentes en el mercado reflejan toda la información disponible. En consecuencia, si el mercado es eficiente, los precios cambiarán cuando se incorpore información no anticipada (Duarte et al., 2014), lo que impedirá la predicción del comportamiento futuro del mercado, debido a que la información nueva que pueda generarse ya se ha reflejado en el precio del activo.

Luego, la hipótesis fuerte de los mercados eficientes, no solo considera la incorporación de información de manera inmediata (Peters, 1989), sino que además los precios podrían manifestar característica de información privilegiada (Cuthbertson y Nitzsche, 2004). Según Merkens (2007), el hecho de que los rendimientos de activos en un mercado respondan a procesos persistentes, refleja la utilización de información privilegiada. Según Peters (1989), lo anterior significa que la incorporación de nuevos antecedentes sobre los retornos es impredecible, y esto permite deducir que el precio actual es relevante en cuanto a su capacidad de predecir comportamientos futuros del mismo precio. Como puede observarse en la literatura, muchos activos no cumplen el supuesto de independencia y, en consecuencia, la existencia de memoria de largo plazo en una serie financiera invalida la hipótesis de mercado eficiente (Rodríguez, 2011) y la información recién incorporada no se reflejará de manera automática en los precios de los activos transados.

En este sentido, Peters (1991) introduce la Hipótesis de Mercados Fractales (HMF) para describir procesos complejos manteniendo su relación con el todo (propiedad de los fractales conocida como autosimilitud). La hipótesis de fractalidad de sucesos naturales (Mandelbrot, 1982) implica esta propiedad de autosimilitud, en el sentido de que las partes responden en una escala menor al todo. Una explicación sobre la dinámica del mercado, es decir, de los movimientos diarios y de los eventos acaecidos, se puede obtener a través del análisis de la autosimilitud combinada con métodos estadísticos cuantitativos. En este sentido, la fractalidad puede ser determinística o estocástica, y en este último caso, un ejemplo de ello serían las series temporales de rendimientos accionarios.

En términos comparativos, la Hipótesis de Mercados Fractales basa su teoría en el uso de la información que cada inversionista le da, según su horizonte temporal, lo que se contrapone a la Hipótesis de Mercado Eficiente, donde es la nueva información incorporada al mercado el soporte de esta teoría. Para Peters (1994), un mercado fractal se comporta de manera ineficiente frente a la existencia de memoria de largo plazo en la serie de tiempo, y además porque presenta ciclos de mercado y equilibrios de carácter temporal (Bilel y Nadhem, 2009; Duarte et al., 2014).

Por tanto, si la HMF es correcta, se podría afirmar que los ciclos económicos y financieros presentan "memoria de largo plazo", mayor a la detectada por los Modelos Autorregresivos $\left(\mathrm{AR}_{\mathrm{n}}\right)$. Por lo anterior, detectar el comportamiento de tales ciclos, implicaría poder predecir el comportamiento de portafolios de inversión, y obtener resultados anormales favorables. Peters (1989) en su trabajo Fractal Structure in the Capital Markets, fue uno de los primeros en estudiar la persistencia e ineficiencia del mercado accionario americano. Su investigación se enfocó en el comportamiento del índice estadounidense S\&P500, cuyo análisis fue por medio del cálculo del coeficiente de Hurst (H). En 1994, Peters formaliza esta metodología a modo de comprobación de la Hipótesis de Mercados Fractales. Otros estudios, como los de Holton (1992) y León y Reveiz (2010), por mencionar algunos, muestran que una 
consecuencia de la memoria de largo plazo (dependencia serial de largo plazo), es que al presentarse en una serie temporal, invalida los supuestos de movimiento browniano (MB). Por otro lado, los estudios de Peters (1989) y de Lo (1991), presentan evidencias teóricas sobre la violación de la HME.

Long term serial dependence

\section{Rango reescalado y coeficiente de Hurst}

Las investigaciones y estudios sobre el concepto de la "memoria de largo plazo" (dependencia serial de largo plazo) comenzaron a desarrollarse a partir del análisis de algunos fenómenos naturales. El físico Harold Edwin Hurst, encontró que lo flujos del río Nilo presentaban memoria de largo plazo, es decir, que el comportamiento de los flujos del río Nilo, se caracterizaba por ser un proceso aleatorio sesgado, presencia de una influencia en futuros acontecimientos por parte de comportamientos pasados (Rodríguez, 2011). El comportamiento anterior también fue encontrado por Mandelbrot (1972) en las series financieras, es decir, dichas series presentaban memoria de largo plazo, lo que se traduce en que los retornos de un activo hoy podrían influir en el comportamiento de los retornos futuros de dicho activo.

En el año 1962, cuando Mandelbrot retomó los estudios de Hurst, desarrolló una generalización de dichas ideas, las que denominó "Rango Reescalado (R/S)". A partir de la propuesta de Hurst (1951), varios autores (Mandelbrot y Wallis, 1969; Mandelbrot, 1972) han empleado el análisis del rango reescalado para contrastar la dependencia a largo plazo en una serie de tiempo, y por el cual Mandelbrot encontró un valor aproximado al exponente de Hurst, al aplicar lo propuesto por Hurst a series de tiempo en campos como Meteorología y Finanzas.

Este análisis del rango reescalado, permite a los investigadores encontrar el coeficiente de Hurst, a través de una metodología que consiste en medir en primera instancia, el rango (R) de la desviación acumulada de la serie $\mathrm{x}_{\mathrm{j}}$ frente a su valor promedio $\mathrm{x}$ « $_{\mathrm{n}}$ durante un período n; es decir, el comportamiento de la serie, luego de excluir el efecto de tendencia, a través de la siguiente expresión matemática:

$$
\mathrm{Rn}=\left[\left(\operatorname{máx}_{1 \leq \mathrm{k} \leq \mathrm{n}}\left(\sum_{\mathrm{j}=1}^{\mathrm{k}}\left(\mathrm{x}_{\mathrm{j}}-\overline{\mathrm{x}}\right)\right)-\operatorname{mín}_{1 \leq \mathrm{k} \leq \mathrm{n}}\left(\sum_{\mathrm{j}=1}^{\mathrm{k}}\left(\mathrm{x}_{\mathrm{j}}-\overline{\mathrm{x}}\right)\right)\right]\right.
$$

Dado que el rango de la serie depende del tiempo, por lo cual el valor de R aumenta toda vez que n lo hace, Hurst estandarizó la medida dividiendo R por la desviación estándar de las observaciones (S):

$$
S_{n}=\left[\frac{1}{n} \sum_{j=1}^{n}\left(x_{j}-\bar{x}\right)^{2}\right]^{1 / 2}
$$

Lo anterior, le permitió a Hurst establecer una ley, conocida como la Ley Empírica de Hurst, y que permite determinar el valor de (R/S) [ver la ecuación (4)]:

$$
\mathrm{R} / \mathrm{S}=\left(\frac{1}{\mathrm{~S}_{\mathrm{n}}}\right)\left[\left(\operatorname{máx}_{1 \leq \mathrm{k} \leq \mathrm{n}}\left(\sum_{\mathrm{j}=1}^{\mathrm{k}}\left(\mathrm{x}_{\mathrm{j}}-\overline{\mathrm{x}}\right)\right)-\operatorname{mín}_{1 \leq \mathrm{k} \leq \mathrm{n}}\left(\sum_{\mathrm{j}=1}^{\mathrm{k}}\left(\mathrm{x}_{\mathrm{j}}-\overline{\mathrm{x}}\right)\right)\right]\right.
$$




$$
\left(\frac{\mathrm{R}}{\mathrm{S}}\right)_{\mathrm{t}}=\mathrm{c} \times \mathrm{n}^{\mathrm{H}}
$$

En la ecuación (4), $\mathrm{H}$ representa el coeficiente de Hurst, $\mathrm{c}$ una constante y n es el período de tiempo. Finalmente, el coeficiente o exponente de Hurst $(\mathrm{H})$, es posible determinarlo por medio de la ecuación (5), luego de aplicar el logaritmo:

$$
\operatorname{LN}\left(\frac{\mathrm{R}}{\mathrm{S}}\right)_{\mathrm{n}} \operatorname{Ln}(\mathrm{c})+\mathrm{H} \times \operatorname{LN}(\mathrm{n})
$$

El coeficiente o exponente de Hurst es un índice mediante el cual se establece la independencia de las series de tiempo, como elemento para distinguir series fractales, y representa un valor numérico que posibilita la determinación de la autocorrelación en una serie de tiempo. Sus valores se encuentran en el rango de (0,1) (Mandelbrot, citado por Espinosa, 2007). Si el valor de $\mathrm{H}$ se encuentra entre $0,5<\mathrm{H}<1$, la serie de tiempo evidencia un proceso persistente o correlacionado, lo que significa que un período de crecimiento es altamente probable que sea seguido por otro período de crecimiento, y viceversa. Lo anterior, es una característica de los efectos de memoria de largo plazo. Por su parte, un valor de $\mathrm{H}$ perteneciente al rango $0<\mathrm{H}<$ 0.5 implica un comportamiento antipersistente o anticorrelacional, lo que significa que un período de crecimiento es altamente probable que sea seguido por un período en decrecimiento y viceversa. Este valor, conocido como "Ruido Rosa", se caracteriza por su alta frecuencia, y por tanto, tiende a regresar a su lugar de procedencia, siendo señales muy irregulares.

Existen dos casos especiales para el valor de $\mathrm{H}$. Cuando el coeficiente $\mathrm{H}=0.5$, conocido como "Ruido Blanco", implica que el proceso es completamente aleatorio e independiente, con una correlación ausente entre los incrementos de la serie, por lo que los retornos de hoy no influyen sobre los retornos futuros. Por otro lado, un valor de $\mathrm{H}=1$, conocido también como "Ruido Negro", indicaría un comportamiento determinístico.

No obstante lo anterior, el comportamiento del coeficiente de Hurst aplicado a una serie independiente presenta una aproximación al valor 0.5 (Rodríguez, 2011) y cuando se utilizan series finitas dicho coeficiente puede resultar mayor a 0.5 .

Coeficiente de Hurst ajustado ( $H_{\text {adi }}$ )

Tal como fue mencionado en el apartado anterior, al utilizar series temporales finitas, se origina un sesgo en la estimación del coeficiente de Hurst (León y Vivas, 2010; León y Reveiz, 2010; Peters, 1994), característica principalmente dada por la naturaleza asintótica del coeficiente.

Para tal efecto, se han propuesto y realizado modificaciones a la metodología de estimación del rango reescalado, lo que permite ajustar el cálculo del coeficiente de Hurst (Peters, 1994; Ellis, 1987; Couillard y Davison, 2005; Ellis, citado por León y Vivas, 2010). Esta propuesta fue desarrollada por Anis y Lloyd (1976) y Peters (1994), y permitiría evitar la sobreestimación del valor del distanciamiento presente con relación a la hipótesis nula de independencia. La ecuación (6) permite el cálculo del coeficiente de Hurst ajustado:

$$
\mathrm{H}_{\mathrm{adj}}=\mathrm{H}-\left(\mathrm{H}_{\mathrm{f}}-0.5\right)
$$

Donde:

$\mathrm{H}_{\text {adj: }}$ Coeficiente Hurst ajustado.

H: Coeficiente Hurst. 
El valor del coeficiente $\mathrm{H}_{\mathrm{f}}$, se obtiene por medio de la esperanza matemática del rango reescalado $\mathrm{E}(\mathrm{R} / \mathrm{S})_{\mathrm{n}}$ para un grupo de datos independiente de tamaño de la muestra (n). Esta esperanza matemática en dicho valor presenta un sesgo positivo, que crece inversamente proporcional al tamaño de dicha muestra de datos, y su estimación se realiza por medio de la ecuación (7) (Anis y Lloyd, Peters, Weron, Couillard y Davison: citados por León y Vivas, 2010):

$$
\mathrm{E}\left(\frac{\mathrm{R}}{\mathrm{S}}\right)_{\mathrm{t}}=\left[\frac{\mathrm{n}-\frac{1}{2}}{\mathrm{n}} \times \frac{1}{\sqrt{\frac{n \pi}{2}}} \times \sum_{\mathrm{i}=1}^{\mathrm{n}-1} \sqrt{\frac{\mathrm{n}-\mathrm{i}}{\mathrm{i}}}\right]
$$

Luego, la estimación del coeficiente $\mathrm{H}_{\mathrm{f}}$, se obtiene por medio de una regresión lineal teniendo como base la estructura de la ecuación (5), como variable independiente LN (n) y como variable dependiente $\mathrm{LN}\left(\mathrm{E}(\mathrm{R} / \mathrm{S})_{t}\right)$, tal como se muestra en la ecuación (8):

$$
\operatorname{LNE}\left(\frac{R}{S}\right)_{t}=\mathrm{LN}(\hat{\mathrm{c}})+\mathrm{H}_{\mathrm{f}} \times \mathrm{LN}(\mathrm{n})
$$

Luego, la estimación final del coeficiente de Hurst ajustado $\left(\mathrm{H}_{\mathrm{adj}}\right)$, se obtiene por medio de la ecuación (6).

\section{Metodología de la investigación}

Por medio de la metodología precisada a continuación, se presentará un análisis del mercado bursátil de Chile, a través de una serie temporal del Índice de Precios Selectivo de Acciones (IPSA) en dos escalas (diaria y trimestral), en un período de 12 años, comprendido entre enero de 2002 y diciembre de 2014. Ello implica examinar la existencia de persistencia e indicios de estructuras caóticas en la serie de tiempo del IPSA y de su rendimiento.

En primer lugar, se desarrolló un breve análisis del mercado, representado por el índice IPSA según metodología de Box y Jenkings. Considerando que los procesos caóticos presentan entre sus características la ausencia de normalidad y la presencia de no-linealidad, será el punto inicial para detectar la presencia o no de estructuras lineales en la serie. En este caso, el análisis se realizó por medio de la observación del comportamiento de la serie gráficamente, y de manera adicional si existen períodos determinados en donde se concentren las principales variaciones del índice, como también si hay evidencia de heteroscedasticidad y variaciones alejadas de la media.

Para validar los resultados del punto anterior, se aplicó el test desarrollado por Brock, Dechert y Scheinkman en 1987 (BDS) sobre los residuos del modelo regresivo estimado. Este test es no-paramétrico, cuya hipótesis nula declara que la serie es independiente e idénticamente distribuida. En este sentido, si el test rechaza la hipótesis nula, se aceptará que la serie presenta estructura no lineal. El procedimiento para ello será través de la aplicación de filtros lineales (ARIMA) y filtros no lineales (GARCH), para luego explorar la remanencia de estructura en los residuos de tales modelos mediante el test BDS.

En segundo lugar se procedió a analizar la dinámica y patrones del índice IPSA y de su rendimiento, para observar si existe evidencia de memoria de largo plazo. Lo anterior, por medio del análisis reescalado, a través de la estimación del coeficiente de Hurst, de manera de contrastar los resultados de los puntos anteriores a partir de una técnica no paramétrica y que contempla la presencia de "memoria de largo plazo": Metodología de rango reescalado.

La serie temporal analizada tiene las siguientes características: cotización diaria y trimestral del índice IPSA de la Bolsa de Comercio de Santiago. Para el caso de valores diarios, se consideró su valor de cierre, y su respectivo rendimiento calculado como el ln 
JEFAS

22,42

44
Tabla I.

Prueba de

Normalidad:

Kolmogorov-

Smirnov (Corrección de la significancia de Lilliefors) (logaritmo natural) del cociente entre el valor del índice del día y el valor del índice para el día anterior (Peters, 1991). Se omitieron los días en los que la Bolsa no operó y los que presentaron valores nulos. Para los valores trimestrales, se consideró el valor promedio del IPSA del subperíodo en estudio.

Las series se analizaron en el período total, y en subperíodos de igual número de observaciones cada uno; el propósito es observar la evolución de la eficiencia del mercado bursátil a través del tiempo. El período de la muestra está comprendido entre el 3 de enero de 2002 y el 31 de diciembre de 2014 (3240 observaciones).

Todos los testeos "tradicionales" (modelos ARIMA, test BDS y coeficiente de Hurst) se aplicaron sobre la variación en términos logarítmicos.

Los softwares utilizados para realizar los cálculos y estimaciones estadísticas fueron IBM SPSS Statistics 20 y Microsoft Excel 2010.

\section{Desarrollo del tema}

A continuación se presentan los resultados de la investigación. En primera instancia, se muestran los cálculos de algunos estadígrafos que permitirán inferir el cumplimiento de condiciones necesarias para determinar la presencia de comportamiento caótico por parte de la serie bursátil, lo cual se presenta de manera cuantitativa y gráfica. Luego se determinan los valores del coeficiente de Hurst y Hurst ajustado para dos escalas de tiempo, con el propósito de deducir la presencia de memoria de largo plazo en la serie de tiempo bursátil.

\section{Normalidad}

Para contrastar la hipótesis de normalidad se utilizaron las pruebas Kolmogorov-Smirnov para la seria diaria y de Shapiro-Wilk para la serie trimestral.

La Tabla I, muestra los resultados de valores P (Sig. Asintót (bilateral)) de la prueba de normalidad de Kolmogorov-Smirnov para los valores diarios del IPSA (Valor IPSA-D), rendimientos diarios del IPSA (Rend IPSA-D) y serie logarítmica de los rendimientos del IPSA (LN Rend IPSA-D), a un 95per cent de confianza.

\begin{tabular}{lccc}
\hline & Valor IPSA-D & Rend IPSA-D & LN Rend IPSA-D \\
\hline Número Datos (N) & 3240 & 3240 & 3240 \\
Estadístico & 0.102 & 0.059 & 0.060 \\
Curtosis & -1.280 & 10.779 & 9.632 \\
Z de Kolmogorov-Smirnov & 5.785 & 3.345 & 3.420 \\
Sig. Asintót (bilateral) & 0.000 & 0.000 & 0.000
\end{tabular}

Fuente: Elaboración propia

\begin{tabular}{lcc}
\hline & Valor IPSA-T & LN Rend IPSA-T \\
\hline Número Datos (N) & 50 & 50 \\
Estadístico & 0.945 & 0.972 \\
Curtosis & -1.271 & -0.481 \\
Sig. & 0.022 & 0.272
\end{tabular}

Tabla II.

Prueba de Normalidad: Shapiro-Wilk

Fuente: Elaboración propia 
La Tabla II muestra los resultados de la prueba de normalidad para las series trimestrales del IPSA. En este caso, dada la cantidad de observaciones (50 datos), la prueba correspondiente es Shapiro-Wilk: valores trimestrales del IPSA (Valor IPSA-T), rendimiento trimestral del IPSA (Rend IPSA-T) y logarítmica de los rendimientos trimestrales del IPSA (LN Rend IPSA-T).

Long term serial dependence

\section{No-linealidad}

Para probar la no-linealidad de las series de este estudio, se aplicó la prueba desarrollada por Brock, Dechert y Scheinkmann (prueba BDS) en 1987. Dado que las series de tiempo no tienen una distribución normal, se espera que los procesos sean no-lineales y que exista una dinámica caótica en ellas. Los valores obtenidos se observan en la Tabla III.

\begin{tabular}{lccccccc}
\hline & \multicolumn{2}{c}{ Dim $=2$} & \multicolumn{2}{c}{ Dim $=3$} & \multicolumn{2}{c}{ Dim $=4$} \\
& $\varepsilon / \sigma$ & z-Statistic & Prob & z-Statistic & Prob & z-Statistic & Prob \\
\hline Valor IPSA-D & $0.5 / \sigma$ & 25.0954 & 0.000 & 37.0078 & 0.000 & 47.8793 & 0.000 \\
& $1 / \sigma$ & 24.0765 & 0.000 & 31.3527 & 0.000 & 36.5654 & 0.000 \\
& $1.5 / \sigma$ & 23.0135 & 0.000 & 27.0216 & 0.000 & 29.8571 & 0.000 \\
LN Rend IPSA-D & $2 / \sigma$ & 21.9754 & 0.000 & 24.0273 & 0.000 & 25.8124 & 0.000 \\
& $0.5 / \sigma$ & 12.4181 & 0.000 & 14.6356 & 0.000 & 16.8634 & 0.000 \\
& $1 / \sigma$ & 12.9687 & 0.000 & 15.7148 & 0.000 & 17.5788 & 0.000 \\
& $1.5 / \sigma$ & 14.1258 & 0.000 & 17.1765 & 0.000 & 18.9754 & 0.000 \\
& $2 / \sigma$ & 14.9012 & 0.000 & 17.8976 & 0.000 & 19.5421 & 0.000
\end{tabular}

Fuente: Elaboración propia
Tabla III.

Estimación del Estadígrafo BDS para las series IPSA diario

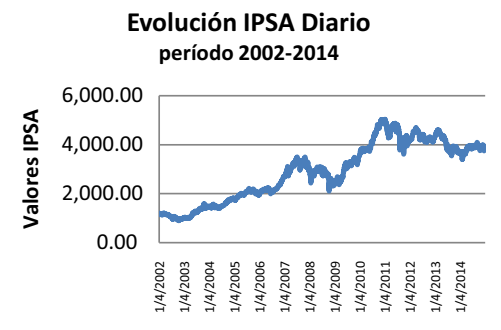

Evolución Rendimiento diario del IPSA período 2002-2014

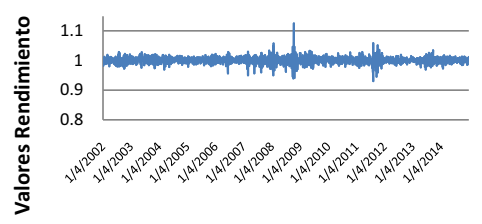

Evolución IPSA Trimestral período 2002-2014

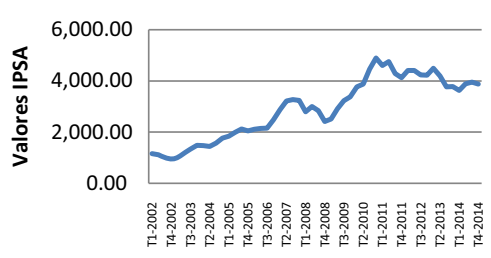

Evolución Rend. trimestral del IPSA período 2002-2014

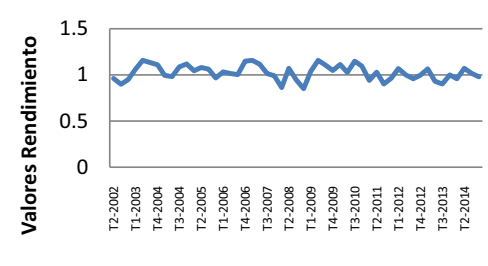

Figura 1. Evolución IPSA, diario y trimestral. Fuente: Elaboración propia
Figura 2.

Evolución rendimientos del IPSA, diario y trimestral. Fuente: Elaboración propia 


\section{JEFAS \\ 22,42}

Tabla IV.

Exponente de Hurst para la serie diaria y trimestral del IPSA

\section{Autosimilitud}

La Autosimilitud es una característica propia de los fractales, donde cada uno de los elementos participantes tiene la capacidad de similitud con respecto al total. Por lo tanto, un comportamiento cercano a este fenómeno se presenta en las Figuras 1 y 2, que muestran las evoluciones del índice diario y trimestral, tanto para el valor del IPSA como para su rendimiento, con el propósito de identificar la presencia de algún patrón similar entre ambas series.

\section{Persistencia y coeficiente de Hurst}

Los resultados relacionados con el cálculo del Exponente de Hurst para la serie original y la serie calculada en escala trimestral de tiempo, aparecen en la Tabla IV.

\section{Coeficiente de Hurst ajustado}

Para identificar la existencia y grado de sesgo posible en el coeficiente de Hurst, dado que se ha trabajado con una serie temporal finita, se calcula el coeficiente de Hurst ajustado $\left(\mathrm{H}_{\mathrm{adj}}\right)$ para los valores originales y su respectivo rendimiento, en ambas escalas de tiempo, cuyos resultados se muestran en la Tabla V. Además, se determina el grado de ajuste (o diferencia) entre el coeficiente de Hurst y Hurst ajustado para los valores del rendimiento del IPSA, resultados que se muestran en la Tabla VI.

\begin{tabular}{lcclcc}
\hline $\begin{array}{l}\text { Valor IPSA } \\
\text { Serie }\end{array}$ & Exp. Hurst & Datos & $\begin{array}{l}\text { Rendimientos del IPSA } \\
\text { Serie }\end{array}$ & Exp. Hurst & Datos \\
\hline Valor IPSA-D & 0.92045 & 3240 & Valor Rend IPSA-D & 0.59857 & 3240 \\
Valor IPSA-T & 1.00000 & 50 & Valor Rend IPSA-T & 0.72086 & 50
\end{tabular}

Fuente: Elaboración propia
Tabla V.

Exponente de Hurst ajustado (Hadj) para la serie diaria y trimestral del IPSA

\begin{tabular}{lcclccr}
\hline $\begin{array}{l}\text { Valor IPSA } \\
\text { Serie }\end{array}$ & Exp. Hurst $_{\text {adj }}$ & Datos & Serie & Exp. Hurst adj & Datos & $H_{f}$ \\
\hline Valor IPSA-D & 0.894356 & 3240 & Valor Rend IPSA-D & 0.572476 & 3240 & 0.526094 \\
Valor IPSA-T & 0.842053 & 50 & Valor Rend IPSA-T & 0.562911 & 50 & 0.657948
\end{tabular}

Fuente: Elaboración propia

\section{Tabla VI.}

Ajuste entre el exponente de Hurst y

Hurst ajustado (Hadj) Valor Rend IPSA-D para los rendimientos Valor Rend IPSA-T de las series diaria y trimestral del IPSA

\begin{tabular}{llll}
\hline & \multicolumn{2}{c}{ Rendimientos del IPSA } & \\
Serie & Exp. Hurst & Exp Hurst adj $\left(\mathrm{H}_{\mathrm{adj}}\right)$ & Ajuste (Diferencia) \\
\hline Valor Rend IPSA-D & 0.59857 & 0.572476 & 0.026094 \\
Valor Rend IPSA-T & 0.72086 & 0.562911 & 0.157949
\end{tabular}

Fuente: Elaboración propia 


\section{Discusión}

Los análisis iniciales que permitirían dar cuenta de indicios de procesos caóticos, normalidad y no-linealidad, muestran que todas las series (Valor IPSA-D, Rend IPSA-D y LN Rend IPSA-D) no cumplen con la hipótesis de normalidad, en un nivel de confianza del 95 per cent (Sig. Asintót-bilateral $=0.000$ ), lo que además se puede comprobar con el coeficiente de curtosis de cada serie analizada (ver la Tabla I).

Por otro lado, como puede observarse en la Tabla II, solo la serie de los valores trimestrales del IPSA (Sig.valor IPSA-T $=0.022$ ) no cumplen con la hipótesis de normalidad, en un nivel de confianza del 95per cent. Lo contrario ocurre con las series asociadas a los rendimientos (Rend IPSA-T y LN Rend IPSA-T) que responden a un comportamiento normal en su distribución.

Al observar las Figuras 1 y 2, los valores y rendimientos del índice muestran que la serie trimestral analizada sigue patrones y tendencias similares a la serie diaria; es decir, el comportamiento evolutivo del índice en una escala de tiempo diferente se comporta como la serie original, el todo. Estos resultados se pueden considerar como una aproximación inicial al fenómeno de autosimilitud. Por tanto, es posible aseverar que los contrastes determinados muestran evidencia de que los procesos de las series temporales analizadas son no-normales y no-lineales, los que teóricamente se consideran una condición necesaria para la existencia de una dinámica caótica.

Recordando la metodología de rango reescalado propuesta por Hurst para la determinación de su coeficiente, es posible observar en ambas series de la Tabla IV los valores del IPSA y rendimiento del IPSA, en sus dos escalas, donde el valor calculado es mayor de 0.5; entonces se puede afirmar que ambas series son persistentes, lo que significa que ambas presentan memoria de largo plazo. Estos resultados son similares a los obtenidos por Peters (1989) para el caso norteamericano y por Hurst para varios fenómenos naturales.

Teniendo en cuenta los resultados, la Hipótesis de Mercado Eficiente se rechaza para la serie temporal del IPSA analizada, por cuanto se comprueba la existencia de persistencia de largo plazo, por lo que, según Peters (1989), hay evidencia de caminata aleatoria sesgada hasta que un evento externo o exógeno ocurra y genere un cambio en el sesgo presente. Esto es señal de existencia de factores que propician la persistencia, tales como noticias y/o anuncios por parte de agentes participantes del mercado bursátil chileno, traducido en la no incorporación inmediata del efecto de dichos eventos en el precio de las acciones y, por lo tanto, el mercado bursátil manifiesta un comportamiento sesgado de largo plazo con características caóticas.

Entonces, si la hipótesis de fractalidad (HF) es correcta, los resultados del coeficiente de Hurst obtenidos permiten pensar que la memoria de largo plazo presente en el indicador del IPSA, es mucho mayor que las que normalmente detectan los modelos autorregresivos $\left(\mathrm{AR}_{\mathrm{n}}\right)$.

Un punto interesante de destacar es que en ambas escalas la serie correspondiente al valor del IPSA muestra un coeficiente de Hurst muy cercano a la unidad, lo que implicaría que el comportamiento de la serie es prácticamente determinístico, específicamente en el caso de la escala trimestral (Ruido Negro), debido a su relación con procesos cíclicos de largo plazo.

Por otro lado, y considerando el ajuste propuesto por Anys y Lloyd (1976) y Peters (1994), el coeficiente de Hurst estimado respalda la visión de presencia de memoria de largo plazo, evitando la sobreestimación con relación a la hipótesis nula de independencia en la serie del IPSA, al resultar con valores superiores a 0.5 en todos sus casos y escalas (ver la Tabla V). Lo anterior se confirma al determinar el grado de ajuste (o diferencia) entre los coeficientes 
JEFAS

22,42

48

de Hurst y Hurst ajustado, cuyo valor es positivo. Cabe destacar que un mayor ajuste se produce en los valores del rendimiento trimestral del IPSA (Valor Rend IPSA-T), porque dicho valor es resultado del promedio aritmético de la serie diaria original para un período cronológico de tres meses, lo que produce una mayor suavidad de los picos en la serie sin modificar la tendencia de la serie misma (ver la Tabla VI). Quiere decir que ambas series, IPSA y rendimientos del IPSA, en sus dos escalas (diaria y trimestral) presentan persistencia de largo plazo y sesgo en su comportamiento, lo que es predecible al tratarse del cálculo considerando una serie finita, que puede causar muchas fluctuaciones estadísticas. Sin embargo, dicho sesgo al ser determinado y eliminado del coeficiente de Hurst, no evita que las series dejen de ser persistentes en cuanto a su memoria de largo plazo (valor $H_{\text {adj }}>0.5$ ) dado que la variable ajuste $H_{f}$ es inferior a dicho coeficiente en ambas series del rendimiento del IPSA como esperanza matemática $\left(H_{f}=0.526094<0.59857 \mathrm{y} H_{f}=0.657948<0.72086\right)$; es decir, esto puede interpretarse como que el mercado no tiene la suficiente madurez y capacidad para traspasar de manera inmediata información a los precios de acciones, dado algún evento caótico en el mismo, manifestándose entonces un sesgo en la serie.

\section{Conclusiones}

En este estudio se han utilizado las técnicas habitualmente usadas para detectar dependencia de largo plazo en series financieras. Los resultados encontrados demuestran la presencia de memoria de largo plazo en el mercado bursátil chileno, determinado a través de índice accionario IPSA en dos escalas, diaria y trimestral, lo que además corrobora los hallazgos de varios autores sobre la utilización de la metodología de rango reescalado para la identificación y determinación de memoria de largo plazo en una serie temporal.

Por medio de la metodología utilizada, es posible evidenciar la presencia de persistencia significativa en el rendimiento del índice bursátil, en sus dos escalas (Hurst valor Rend IPSA-D = 0.59857 y Hurst valor Rend IPSA-T $=0.72086$ ), lo que además se confirma al ajustar el coeficiente de Hurst $\left(\mathrm{H}_{\mathrm{adj}}\right)$ para dicho índice en sus dos escalas, obteniéndose valores superiores a 0.5; indicativo de que las series temporales no siguen un comportamiento aleatorio o de Ruido Blanco. Con lo anterior se ha evitado una sobreestimación del valor del distanciamiento presente respecto de la hipótesis nula de independencia, especialmente en la serie temporal a escala trimestral, donde se ha producido un mayor ajuste (diferencia) entre ambos cálculos del coeficiente de Hurst (ajuste $=0.157949$ ).

En este sentido, el cálculo de dicho ajuste, ha permitido discriminar entre el rechazo o aceptación de la hipótesis nula de independencia de largo plazo en la serie temporal, al utilizarse una serie finita para dicho estudio. Es probable que la comprobación sobre las características de no-normalidad y no-linealidad, necesarias para comprobar la presencia de una dinámica caótica, se realice por medio de la dimensión fractal. Por otro lado, requerirá de la incorporación de información del test $\mathrm{ARCH}$, para evidenciar en contraposición la presencia de este efecto después de filtrar las series con un modelo ARIMA (p, d, q).

Dadas las características del mercado bursátil chileno, los factores exógenos que propiciarían el comportamiento sesgado de dicho mercado podrían estar relacionados con alguna de las siguientes variables:

- información privilegiada, es decir, si se dan las condiciones de existencia de esta información, se facilita su uso;

- marco regulatorio que rige en este mercado, facilitaría las condiciones de imitación de algunas conductas por parte de algunos agentes participantes; y 
- información, la que al ser escasa o incluso ausente, también facilitaría la imitación de conductas, apoyada incluso por el marco regulatorio existente.

Long term serial dependence

\section{Referencias}

Anis, A.A. y Lloyd, E.H. (1976), "The expected value of the adjusted rescaled Hurst Range of independent normal summands", Biometrika, Vol. 63 No. 1, pp. 111-116.

Bilel, T.Y. y Nadhem, S. (2009), "Long memory in stock returns: Evidence of G7 stock markets", Research Journal of International Studies, Vol. 9, pp. 36-46.

CEPAL (2011), "La inversión extranjera directa en América Latina y el Caribe 2010, 2011", available at: www.cepal.org/es/publicaciones/1141-la-inversion-extranjera-directa-en-america-latina-yel-caribe-informe-2010 (accessed 11 February 2015).

Couillard, M. y Davison, M. (2005), "A comment on measuring the hurst exponent of financial time series”, Physica A: Statistical Mechanics and its Applications, Vol. 348 No. 1, pp. 404-418.

Cuthbertson, K. y Nitzsche, D. (2004), Quantitative Financial Economics, JohnWiley y Sons.

Davies, R. y. Harte, D. (1987), “Test for Hurst effect”, Biometrika, Vol. 74 No. 1, pp. 95-101.

Duarte, J., Sierra, K., Pérez-Iñigo, M. y Manuel, J. (2014), “Evaluación de la memoria de largo plazo del mercado bursátil colombiano mediante el coeficiente de Hurst", Revista Internacional Administración y Finanzas, Vol. 7 No. 4, pp. 1-10.

Ellis, C. (1987), "The sampling properties of Hurst exponent estimates", Physica A: Statistical Mechanics and its Applications, Vol. 375 No. 1, pp. 159-173.

Espinosa, C. (2007), "Memoria de largo plazo y efecto reset en retornos accionarios latinoamericanos", Estudios de Administración, Vol. 14 No. 1, pp. 47-70.

Espinosa, C., Parisi, F. y Parisi, A. (2005), "Evidencia de comportamiento caótico en índices bursátiles americanos", Trimestre Económico, p. 296

Fama, E. (1965), "The behavior of stock-market prices", The Journal of Business, Vol. 38 No. 1, pp.34-105.

Fama, E. y French, K.R. (1988), "Permanent and temporary components of stock prices", Journal of Political Economy, Vol. 96 No. 2, pp. 246-273.

FMI (2010), Aprovechando El Viento a Favor, Perspectivas económicas, Las Américas.

Holton, G. (1992), "Time: the second dimension of risk", Financial Analysts Journal, Vol. 48 No. 6 , pp. 38-45.

Hurst, H.E. (1951), "Long-term storage capacity of reservoirs", Transactions of the American Society of Civil Engineers, Vol. 116, pp. 770-799.

Karuppiah, J. y Los, C. (2005), "Wavelet multiresolution analysis of high frequency asian FX rates", International Review of Financial Analysis, Vol. 14, pp. 211-246.

León, C. y Reveiz, A. (2010), Portfolio Optimization and Long-Term Dependence, Borradores de Economía, Banco de la República, p. 648.

León, C. y Vivas, F. (2010), Dependencia de largo plazo y la regla de la raiz del Tiempo Para Escalar la Volatilidad en el Mercado Colombiano, Borradores de Economía, Banco de la República, p. 603.

Lo, A. (1991), "Long-term memory in stock market prices", Econometrica, Vol. 59 No. 5, pp. 1279-1313.

Lo, A. y Mackinley, C. (1988), "Stock market prices do not follow random walk: evidence from a simple specification test”, Review of Financial Studies, Vol. 1 No. 1, pp. 41-66.

Mandelbrot, B. (1972), "Statistical methodology for nonperiodic cycles: from the covariance to the R/S analysis", Annal of Economic and Social Measuremente, Vol. 1 No. 3, 
JEFAS

22,42

50

Mandelbrot, B. (1982), The Fractal Geometry of Nature, Freeman, San Francisco, CA.

Mandelbrot, B. y Wallis, J.R. (1969), "Robustness of the rescaled range R/S in the measurement of noncyclic long-run statistical dependence", Water Resources Research, Vol. 5 No. 5 , pp. 967-988.

Merkens, O. (2007), “Value at risk and self-similarity”, in Miller, J., Edelman, D. and Appleby, J. (Eds), Numerical Methods for Finance, Chapman.

Parisi, F., Espinosa, C. y Parisi, A. (2007), "Pruebas de comportamiento caótico en índices bursátiles americanos", El Trimestre Económico, Vol. 74 No. 4, pp. 901-927.

Peters, E. (1989), "Fractal structure in the capital markets", Financial Analysts Journal, Vol. 45 No. 4, pp. 32-37.

Peters, E. (1991), Chaos and Order in the Capital Markets: A New View of Cycles, Pricesand Markets Volatily, Jhon Whiley \& Sons, New York, NY.

Peters, E. (1992), "R/S analysis using logarithmic returns: a technical note", Financial Analysts Journal, Vol. 48 No. 6, pp. 81-82.

Peters, E. (1994), Fractal Market Analysis: Applying Chaos Theory to Investment and Economics, John Wiley \& Sons.

Restrepo, J. y Velásquez, H. (2011), “Análisis del índice general de las bolsas de valores de Colombia (IGBC), Chile (IPSA) and Perú (IGBVL), and sus rendimientos desde la teoría del caos 2001-2011", Tesis Maestría en Finanzas, Medellín.

Rodríguez, J.K. (2011), Validez del supuesto de neutralidad del horizonte de tiempo en el CAPM y la metodología del rango reescalado: aplicación de Colombia ( $N^{\circ}$ 009016), Banco de la República.

Sadique, S. y Silvapulle, P. (2001), "Long-term memory in stock market returns: International evidence", International Journal of Finance and Economics, Vol. 6, pp. 59-67.

Soria, B.K. y Zúñiga, J.S. (2004), Algunas Estimaciones del Coeficiente de Hurst Para el IGPA Chileno, Universidad Católica, Escuela de Ingeniería del Norte, Coquimbo.

\section{Corresponding author}

Christian Acuña-Opazo can be contacted at: cacuna@userena.cl

For instructions on how to order reprints of this article, please visit our website: 